\title{
A Novel Approach Based on Decreased Dimension and Reduced Gray Level Range Matrix Features for Stone Texture Classification
}

\author{
G. S. N. Murthy ${ }^{1}$, Srininvasa Rao. $\mathbf{V}^{2}$, T. Veerraju ${ }^{3}$ \\ ${ }^{1}$ Rayalaseema University, Kurnool, India \\ ${ }^{2}$ Dept of CSE, V.R. Siddhartha Engineering College, Vijayawada, India \\ ${ }^{3}$ Dept of CSE, Aditya college of Engineering, Surampalem, India
}

\begin{tabular}{l}
\hline \hline Article Info \\
\hline Article history: \\
Received Mar 31, 2017 \\
Revised Jun 5, 2017 \\
Accepted Sept 11, 2017 \\
\hline
\end{tabular}

\section{Keywords:}

Classification

Co-occurrence matrix

Fuzzy logic

Reduced dimensionality

Stone texture

\begin{abstract}
The human eye can easily identify the type of textures in flooring of the houses and in the digital images visually. In this work, the stone textures are grouped into four categories. They are bricks, marble, granite and mosaic. A novel approach is developed for decreasing the dimension of stone image and for reducing the gray level range of the image without any loss of significant feature information. This model is named as "Decreased Dimension and Reduced Gray level Range Matrix (DDRGRM)" model. The DDRGRM model consists of 3 stages. In stage 1 , each $5 \times 5$ sub dimension of the stone image is reduced into $2 \times 2$ sub dimension without losing any important qualities, primitives, and any other local stuff. In stage 2 , the gray level of the image is reduced from 0-255 to 0-4 by using fuzzy concepts. In stage 3 , Cooccurrence Matrix (CM) features are derived from the DDRGRM model of the stone image for stone texture classification. Based on the feature set values, a user defined algorithm is developed to classify the stone texture image into one of the 4 categories i.e. Marble, Brick, Granite and Mosaic. The proposed method is tested by using the K-Nearest Neighbor Classification algorithm with the derived texture features. To prove the efficiency of the proposed method, it is tested on different stone texture image databases. The proposed method resulted in high classification rate when compared with the other existing methods.
\end{abstract}

Copyright $@ 2017$ Institute of Advanced Engineering and Science. All rights reserved.

\section{Corresponding Author:}

G. S. N. Murthy,

Aditya College of Engineering, Surampalem, Andhra Pradesh, India - 533437.

Email: murthygsnm@yahoo.com

\section{INTRODUCTION}

One of the main contents of the image is texture. The analysis of textures mainly includes: Patterns identified on texture, shapes recovery from texture, Texture segmentation, and texture classification [1]. Among them, texture classification plays an important role in many areas such as remote sensing, stone type identification in construction filed, medical imaging and so on [2]. Texture analysis is one of the prime techniques when applied on images consisting of repetition or quasi repetition of some fundamental image elements. They are analyzed and interpreted by Yagnanarayana [3]. From the past two decades, so many techniques have been identified for texture analysis especially in feature extraction and classification areas. There are so many variations in texture analysis techniques because of the textures have different types of patterns and shapes on the surface. For achieving better performance, different types of features are extracted to characterize the texture images. Texture analysis and investigations are significantly accomplished in one of the two ways, i.e. structural approach and statistical strategy. Structural approach essentially focuses on the stochastic things of the spatial circulation of dark levels in a picture. In finding the characteristics of an 
image, co-occurrence matrix is widely used. From the co-event lattice set of textural elements separated and these components are broadly used to remove textural data from advanced pictures [4][5]. In basic approach, surface is considered as reiteration of a few primitives. For surface grouping and portrayal, these strategies have been connected by a few creators and made progress to a specific degree [6].

So many approaches are available in the literature for texture classification. The first and top most approach is Local Binary Pattern (LBP) approach [7][8]. But LBP approach has some disadvantages. If the central pixel value changes by 1 , the LBP value drastically changes. Other existing approaches are based on wavelet transform [9][10], statistical learning from morphological image processing [11], long linear patterns [12][13], edge direction movements [14], excluding Complex Patterns [15] and preprocessed images [16]. Texture pictures are characterized by utilizing different wavelet transforms using statistical parameters [17] and primitive parameters.

Recently, Juan Wang et.al [18] proposed a method for texture classification using Scattering Statistical and Co-occurrence Features. Wang developed new approach for texture features extraction. This approach used scattering transform for scattering statistical features and scattering co-occurrence features extraction which are derived from sub-bands of the scattering decomposition and original images and these features are used for classification. This approach got reasonable percentage rate of classification but the time complexity is more.

Siva Kumar et.al [19] proposed a method for stone texture classification based on edge direction movement. In this approach, edge movements are identified on each $3 \times 3$ sub-image and based on the edge direction movements, the texture images are classified. This approach mainly classifies the texture image into two groups only and each group consists of 4 different types of texture images. Ratna Bhargavi et al [20] proposed an approach for detection of Lesion using texture features and Xiaorong Xue et.al [21] proposed an approach for Classification of Fully Polari metric SAR Images based on Polari metric Features and Spatial Features.

Vijay Kumar et.al [22] proposed a method for classifying the stone textures into four categories based on occurrence of T-pattern count which are overlapped 5 bit T-patterns on each $5 \times 5$ sub-image. The classification rate of this approach is about 96.16\%. In Vijay Kumar's work, standard classification algorithms are not used for classifying the stone texture group. Standard classification systems consume more time for extraction of the features from stone image and also for classification.

The existing standard classification approaches, both classification of stone textures and extraction of the features from stone image consume more time. Other existing approaches in literature, even proposed algorithms for classifying the stone texture group. Their classification results are not compared with standard classification algorithms to verify the accuracy. If correct features are extracted then they fit for both standard classification and also for user defined algorithm. So, the present work concentrates on developing a method called DDRGRM for classifying the stone textures into four groups.

Till now majority of the existing techniques extract features from the entire image. The proposed DDRGRM strategy is to decrease the stone image dimensionality into $(2 \mathrm{~N} / 5 \times 2 \mathrm{M} / 5)$ and applies fuzzy concept for lessening the dim level range for viable and proficient stone surface grouping. Another fundamental issue in classification of texture and recognition is texture characterization from derived features. Many of the existing approaches have the drawback of computational complexity as they include processing of entire image with large range of gray levels for texture classification and recognition. To address this, the present paper proposes an approach in which the image dimension and dim level range are decreased with no loss of surface component data.

The main objective of the proposed method is to be compatible with both the approaches i.e. for user defined algorithm and also for standard classification algorithms. The proposed method does not use any standard classification algorithms for classifying the stone texture group. The rest of the paper is organized as follows. Section 2 describes the proposed method. Derived user defined algorithm and Results are explained in section 3. Finally, conclusions are given in section 4.

\section{PROPOSED METHOD}

For portraying the attributes of the neighborhood example of the surface by utilizing surface descriptor strategies, for example, Local Binary Pattern (LBP),Texture Unit (TU) and Textons. The surface descriptors are valuable for surface examination and critical grouping and it gives both factual and auxiliary qualities of a surface. These descriptors are totally nearby and generally characterized on a $3 \times 3$ neighborhood. The proposed technique display takes a $5 \times 5$ neighborhood, and reduces it into a $2 \times 2$ neighborhood without loss of any surface data and further it diminishes the dim level range utilizing fluffy rationale. 
The proposed DDRGRM model mainly consists of 6 steps. In step 1, convert the RGB stone texture image into Gray level image using Weighted RGB conversion method. Formation of nine overlapped sub $3 \times 3$ sub images from a $5 \times 5$ sub image is performed in step 2. In step 3 Derivation of "Local Difference Matrix (LDM)" on the nine overlapped $3 \times 3$ sub images and generate the reduced matrix. Further reduce the $3 \times 3$ sub image into $2 \times 2$ sub image without losing the texture image information in step 4 . Step 5, reduce the gray level range in each $2 \times 2$ sub image using fuzzy concept and generate the Fuzzy reduced co-occurrence matrix, in step 6, extract the CM features for classification. The block diagram of the proposed model is shown in Figure 1.

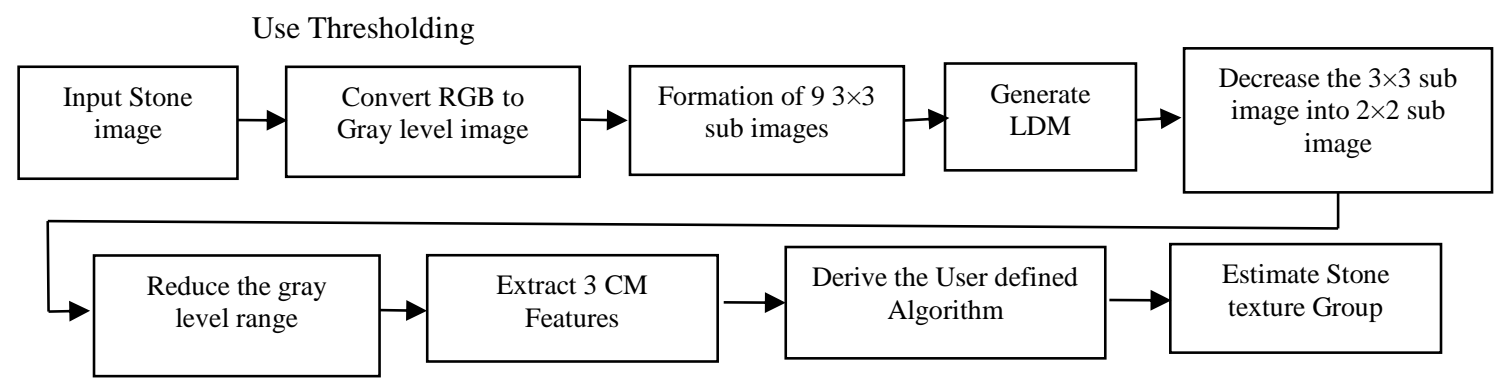

Figure 1. Block diagram of DDRGRM Model

\subsection{Convert RGB to Gray level image:}

To extract the features the RGB image will be transformed to Gray image using Weighted RGB conversion. As the RGB image is formed by 3 commanded hues i.e. Red, Green and Blue, in Weighted RGB conversion diverse weights are assigned to each shading segment and these three segments are used for converting the RGB image to gray image. The transformation procedure is specified in equation 1.

$$
\operatorname{Gray}(x, y)=0.3 * R(x, y)+0.59 * G(x, y)+0.11 * B(x, y)
$$

Where R, G, B are the Red, Green, Blue color component values, (x,y) are the pixel positions and $\operatorname{Gray}(\mathrm{x}, \mathrm{y})$ represents the gray value at the given pixel position $(\mathrm{x}, \mathrm{y})$. The RGB image and resultant gray image after conversion are shown in Figure 2.

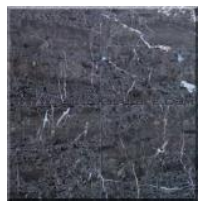

(a)

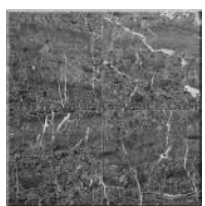

(b)

Figure 2. Marble stone image (a) Color image (b) Resultant Gray level image

\subsection{Formation of 9 overlapped $3 \times 3$ sub images from a $5 \times 5$ sub image:}

The $5 \times 5$ sub image consists of 25 pixels represented by $\{\mathrm{V} 1, \mathrm{~V} 2, \ldots ., \mathrm{V} 13, \ldots \mathrm{V} 25\}$, where V13 represents the gray value of the innermost center pixel and remaining are the neighboring pixel intensity values as shown in Figure 3. Figure 4 represents overlapped $3 \times 3$ sub windows referred as $\{w 1$, w2, w3,... w9 \} extracted from the $5 \times 5$ sub image represented in Figure 3. 


\begin{tabular}{|l|l|l|l|l|}
\hline $\mathrm{V}_{1}$ & $\mathrm{~V}_{2}$ & $\mathrm{~V}_{3}$ & $\mathrm{~V}_{4}$ & $\mathrm{~V}_{5}$ \\
\hline $\mathrm{V}_{6}$ & $\mathrm{~V}_{7}$ & $\mathrm{~V}_{8}$ & $\mathrm{~V}_{9}$ & $\mathrm{~V}_{10}$ \\
\hline $\mathrm{V}_{11}$ & $\mathrm{~V}_{12}$ & $\mathrm{~V}_{13}$ & $\mathrm{~V}_{14}$ & $\mathrm{~V}_{15}$ \\
\hline $\mathrm{V}_{16}$ & $\mathrm{~V}_{17}$ & $\mathrm{~V}_{18}$ & $\mathrm{~V}_{19}$ & $\mathrm{~V}_{20}$ \\
\hline $\mathrm{V}_{21}$ & $\mathrm{~V}_{22}$ & $\mathrm{~V}_{23}$ & $\mathrm{~V}_{24}$ & $\mathrm{~V}_{25}$ \\
\hline
\end{tabular}

Figure 3 . Representation of a $5 \times 5$ sub image

\begin{tabular}{|c|c|c|}
\hline $\mathrm{V}_{1}$ & $\mathrm{~V}_{2}$ & $\mathrm{~V}_{3}$ \\
\hline $\mathrm{V}_{6}$ & $\mathrm{~V}_{7}$ & $\mathrm{~V}_{8}$ \\
\hline $\mathrm{V}_{11}$ & $\mathrm{~V}_{12}$ & $\mathrm{~V}_{13}$ \\
\hline
\end{tabular}

$\mathrm{w}_{1}$

\begin{tabular}{|c|c|c|}
\hline $\mathrm{V}_{6}$ & $\mathrm{~V}_{7}$ & $\mathrm{~V}_{8}$ \\
\hline $\mathrm{V}_{11}$ & $\mathrm{~V}_{12}$ & $\mathrm{~V}_{13}$ \\
\hline $\mathrm{V}_{16}$ & $\mathrm{~V}_{17}$ & $\mathrm{~V}_{18}$ \\
\hline
\end{tabular}

$\mathrm{W}_{4}$

\begin{tabular}{|l|l|l|}
\hline $\mathrm{V}_{11}$ & $\mathrm{~V}_{12}$ & $\mathrm{~V}_{13}$ \\
\hline $\mathrm{V}_{16}$ & $\mathrm{~V}_{17}$ & $\mathrm{~V}_{18}$ \\
\hline $\mathrm{V}_{21}$ & $\mathrm{~V}_{22}$ & $\mathrm{~V}_{23}$ \\
\hline
\end{tabular}

$\mathrm{w}_{7}$

\begin{tabular}{|c|c|c|}
\hline $\mathrm{V}_{2}$ & $\mathrm{~V}_{3}$ & $\mathrm{~V}_{4}$ \\
\hline $\mathrm{V}_{7}$ & $\mathrm{~V}_{8}$ & $\mathrm{~V}_{9}$ \\
\hline $\mathrm{V}_{12}$ & $\mathrm{~V}_{13}$ & $\mathrm{~V}_{14}$ \\
\hline
\end{tabular}

$\mathrm{w}_{2}$

\begin{tabular}{|c|c|c|}
\hline $\mathrm{V}_{7}$ & $\mathrm{~V}_{8}$ & $\mathrm{~V}_{9}$ \\
\hline $\mathrm{V}_{12}$ & $\mathrm{~V}_{13}$ & $\mathrm{~V}_{14}$ \\
\hline $\mathrm{V}_{17}$ & $\mathrm{~V}_{18}$ & $\mathrm{~V}_{19}$ \\
\hline
\end{tabular}

$\mathrm{W}_{5}$

\begin{tabular}{|l|l|l|}
\hline $\mathrm{V}_{12}$ & $\mathrm{~V}_{13}$ & $\mathrm{~V}_{14}$ \\
\hline $\mathrm{V}_{17}$ & $\mathrm{~V}_{18}$ & $\mathrm{~V}_{19}$ \\
\hline $\mathrm{V}_{22}$ & $\mathrm{~V}_{23}$ & $\mathrm{~V}_{24}$ \\
\hline
\end{tabular}

$\mathrm{w}_{8}$

\begin{tabular}{|c|c|c|}
\hline $\mathrm{V}_{3}$ & $\mathrm{~V}_{4}$ & $\mathrm{~V}_{5}$ \\
\hline $\mathrm{V}_{8}$ & $\mathrm{~V}_{9}$ & $\mathrm{~V}_{10}$ \\
\hline $\mathrm{V}_{13}$ & $\mathrm{~V}_{14}$ & $\mathrm{~V}_{15}$ \\
\hline
\end{tabular}

$\mathrm{w}_{3}$

\begin{tabular}{|c|c|c|}
\hline $\mathrm{V}_{8}$ & $\mathrm{~V}_{9}$ & $\mathrm{~V}_{10}$ \\
\hline $\mathrm{V}_{13}$ & $\mathrm{~V}_{14}$ & $\mathrm{~V}_{15}$ \\
\hline $\mathrm{V}_{18}$ & $\mathrm{~V}_{19}$ & $\mathrm{~V}_{20}$ \\
\hline
\end{tabular}

$\mathrm{w}_{6}$

\begin{tabular}{|l|l|l|}
\hline $\mathrm{V}_{13}$ & $\mathrm{~V}_{14}$ & $\mathrm{~V}_{15}$ \\
\hline $\mathrm{V}_{18}$ & $\mathrm{~V}_{19}$ & $\mathrm{~V}_{20}$ \\
\hline $\mathrm{V}_{23}$ & $\mathrm{~V}_{24}$ & $\mathrm{~V}_{25}$ \\
\hline
\end{tabular}

$\mathrm{w}_{9}$

Figure 4. Formation of overlapped $3 \times 3$ neighborhoods $\left\{w_{1}, w_{2}, w_{3}, \ldots, w_{9}\right\}$ from Figure 3

\subsection{Derivation of LDM on each $3 \times 3$ overlapped window of $5 \times 5$ sub image:}

In this step, LDM is figured for every one of the nine $3 \times 3$ covered windows $\{w 1, w 2, w 3, \ldots, w 9\}$ of $5 \times 5$ sub picture. The LDM gives a productive portrayal of surface picture. The LDM on each $w_{i}$ is the outright contrast between the neighboring pixel and the dark estimation of the focal pixel which is evaluated using equation 2 and represented in Figure 5. This results in nine new $3 \times 3$ LDMs represented as \{LDM1, LDM2, LDM3, .., LDM9 $\}$ for each overlapped window $\left\{\mathrm{w}_{1}, \mathrm{w}_{2} \ldots \mathrm{w}_{9}\right\}$.

$$
\mathrm{LDM}_{\mathrm{i}}=\operatorname{abs}\left(\mathrm{v}_{\mathrm{i}}-\mathrm{v}_{\mathrm{c}}\right) \text { for } \mathrm{i}=1,2, \ldots 9
$$

Where $\mathrm{v}_{\mathrm{c}}$ is the centre pixel and $\mathrm{v}_{\mathrm{i}}$ represent the neighboring pixel values of the overlapped $3 \times 3$ neighborhood. Basing on equation 2 the resultant value of each LDM in which the central pixel value is always zero.

\begin{tabular}{|l|l|l|l|l|l|l|l|}
\hline $\mathrm{V}_{1}-\mathrm{V}_{7}$ & & & $\mathrm{~V}_{2}-\mathrm{V}_{7}$ & & & $\mathrm{~V}_{3}-\mathrm{V}_{7}$ & \\
\hline $\mathrm{V}_{6}-\mathrm{V}_{7}$ & & & $\mathrm{~V}_{7}-\mathrm{V}_{7}$ & & & $\mathrm{~V}_{8}-\mathrm{V}_{7}$ & \\
\hline $\mathrm{V}_{11}-\mathrm{V}_{7}$ & & & $\mathrm{~V}_{12}-\mathrm{V}_{7}$ & & & $\mathrm{~V}_{13}-\mathrm{V}_{7}$ & \\
\hline
\end{tabular}

Figure 5. Generation of $\mathrm{LDM}_{1}$ from $\mathrm{w}_{1}$. 


\subsection{Generation of Decrease Dimension Matrix $(D D M)$ of $5 \times 5$ into $3 \times 3$ window:}

In this each value of DDM is evaluated from each of the nine LDM's generated in the previous step in two stages: generation of Mean LDM in the first step and then generate DDM. In stage one, the mean of the 9 windows which are generated in previous step by using the equation 3 are found. The generated values forms a matrix is called Mean LDM (MLDM). The MLDM is a $3 \times 3$ window with nine elements (MLDP ${ }_{1}$ to $\mathrm{MLDP}_{9}$ ). The MLDM preserves the local region possessions including edge information.

$$
\operatorname{MLDP}_{\mathrm{i}}=\text { meanof }\left(\mathrm{LDM}_{\mathrm{i}}\right) \text { for } \mathrm{i}=1,2, \ldots 9
$$

Further, generate the $D D M$ by calculating the local difference between the neighboring pixel values and central pixel value of the MLDP matrix and is represented by equation 4 .

$$
\operatorname{DDMP}_{\mathrm{i}}=\operatorname{abs}\left(\mathrm{MLDP}_{\mathrm{i}}-\mathrm{MLDP}_{\mathrm{c}}\right) \text { for } \mathrm{MLDP}_{\mathrm{i}}=1,2, \ldots 9
$$

The Equation 4 reveals that continuously dominant pixel value of the $3 \times 3 \mathrm{DDM}$ is zero.

\subsection{Generation of Reduced Dimension Matrix (RDM) of $2 \times 2$ window from DDM:}

The generation process of RDM marix is shown in figure 6 . The DDM window comprises of nine qualities which is created in previous step as shown in figure 6(a). In this progression, the DDM of a $3 \times 3$ neighborhood is lessened into a $2 \times 2$ RDM by utilizing Triangular Shape Primitives (TSP). The proposed TSP is an associated neighborhood of three pixels on a $3 \times 3 \mathrm{DDM}$, without focal pixel. The TSP's on DDM doesn't consider focal pixel as its dark level is constantly zero. The normal of these TSP's creates pixel estimations of Reduced Dimension Matrix (RDM) of measure $2 \times 2$ as appeared in Figure 6(b) based on equations 5 to 8 . By this the proposed technique decreases the texture image of size $\mathrm{N} \times \mathrm{M}$ into the size $(2 \mathrm{~N} / 5) \times(2 \mathrm{M} / 5)$.

$$
\begin{aligned}
& \mathrm{RDMP}_{1}=\left(\mathrm{DDMP}_{1}+\mathrm{DDMP}_{2}+\mathrm{DDMP}_{4}\right) / 3 \\
& \mathrm{RDMP}_{2}=\left(\mathrm{DDMP}_{2}+\mathrm{DDMP}_{3}+\mathrm{DDMP}_{6}\right) / 3 \\
& \mathrm{RDMP}_{3}=\left(\mathrm{DDMP}_{4}+\mathrm{DDMP}_{7}+\mathrm{DDMP}_{8}\right) / 3 \\
& \mathrm{RDMP}_{4}=\left(\mathrm{DDMP}_{6}+\mathrm{DDMP}_{8}+\mathrm{DDMP}_{9}\right) / 3
\end{aligned}
$$

\begin{tabular}{|l|l|l|}
\hline DDMP $_{1}$ & DDMP $_{2}$ & DDMP $_{3}$ \\
\hline DDMP $_{4}$ & DDMP $_{5}$ & DDMP $_{6}$ \\
\hline DDMP $_{7}$ & DDMP $_{8}$ & DDMP $_{9}$ \\
\hline
\end{tabular}

(a)

\begin{tabular}{|l|l|}
\hline $\mathrm{RDMP}_{1}$ & $\mathrm{RDMP}_{2}$ \\
\hline $\mathrm{RDMP}_{3}$ & $\mathrm{RDMP}_{4}$ \\
\hline
\end{tabular}

(b)

Figure 6. Generation process of a RDM of size $2 \times 2$ from a $3 \times 3$ DDM neighborhood.
a) The DDM neighborhood
b) RDM.

\subsection{Reduction of gray level range in RDM using fuzzy logic:}

Fuzzy rationale has certain real focal points over conventional Boolean rationale with regards to certifiable applications, for example, surface portrayal of genuine pictures. To deal precisely with the areas of regular pictures even within the sight of clamor and the diverse procedures of subtitle and digitization fluffy rationale is presented on DDM. The proposed fluffy rationale converts DDM dark levels into 5 levels ranging from 0 to 4. The resultant framework is called Decrease Dimension Reducing Gray level Range Matrix (DDRGRM). In LBP double examples are assessed by contrasting the neighboring pixels and focal pixel. The proposed DDRGRM model is determined by looking at the every pixel of the $2 \times 2$ DDM with the normal pixel estimations of the DDM. The DDRGRM portrayal is appeared in Figure 7. The accompanying Equations 9 is utilized to decide the components of DDRGRM model. 


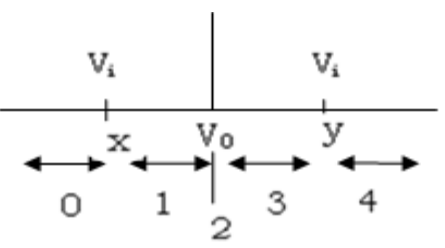

Figure 7. Fuzzy representation of DDRGRM model of the image

$$
\text { DDRGRMP }_{\mathrm{i}}=\left\{\begin{array}{c}
0 \text { ifRDMP }_{\mathrm{i}}<\mathrm{V}_{0} \text { and } \text { RDMP }_{\mathrm{i}}<\mathrm{x} \\
1 \text { ifRDMP }_{\mathrm{i}}<\mathrm{V}_{0} \text { and } \text { RDMP }_{\mathrm{i}} \geq \mathrm{x} \\
2 \text { ifRDMP }_{\mathrm{i}}=\mathrm{V}_{0} \\
3 \text { ifRDMP }_{\mathrm{i}}>\mathrm{V}_{0} \text { and } \operatorname{RDMP}_{\mathrm{i}}>\mathrm{y} \\
4 \text { ifRDMP }_{\mathrm{i}}>\mathrm{V}_{0} \text { and } \operatorname{RDMP}_{\mathrm{i}} \leq \mathrm{y}
\end{array}\right\} \text { for } \mathrm{i}=1,2,3,4
$$

Where $x, y$ are the user-specified values and $\mathrm{V}_{0}=\frac{\left(\sum_{\mathrm{i}=1}^{4} \mathrm{TSP}_{\mathrm{i}}\right)}{\mathbf{4}}$

For example, the process of evaluating DDRGRM model from a sub RDM image of $2 \times 2$ is shown in Figure 8. The Figure 8 (a) represents RDM and figure 8 (b) represents the rsultent fuzzy matrix from RDM. In this study, $\mathrm{x}$ and $\mathrm{y}$ values are chosen as $\mathrm{V}_{0} / 2$ and $3 \mathrm{~V}_{0} / 2$ respectively.

\begin{tabular}{|c|c|}
\hline 28 & 39 \\
\hline 61 & 9 \\
\hline
\end{tabular}

(a)

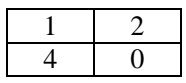

(b)

Figure 8. The process of evaluating DDRGRM model from sub RDM (a) RDM (b) DDRGRM model

\subsection{Computation of CM features on the derived DDRGRM model:}

The present approach determined Gray Level Co-occurrence Matrix (GLCM) on the DDRGRM model of the stone texture image. GLCM is proposed by Haralick to characterize the image based on how certain dark levels happen in comparison with other dim levels. GLCM can gauge the surface of the picture since co-event frameworks are generally vast and scanty. GLCM is considered to be a benchmark for extracting Haralick features like angular second moment, contrast, correlation, variance, inverse difference moment, sum average, sum variance, sum entropy, entropy, difference variance, difference entropy, information measures of correlation and maximal correlation coefficient, etc.. These elements have been broadly utilized as a part of the investigation, grouping and elucidation of picture information. Its point is to portray the stochastic properties of the spatial conveyance of dark levels in an image. Out of these proposed Haralick features the proposed approach used three Haralick highlights i.e. Correlation (CR), Cluster Prominence (CP) and Information measure of correlation1 (IMC1) for classification of stone texturesinto 4 different groups. For characterization of stone textures into 4 unique groups equations (11) to (13) are used. The DDRGRM method with GLCM consolidates the benefits of both statistical and structural information of the stone texture image.

$$
\text { corelation }=\sum_{i=1}^{m} \sum_{j=1}^{n} \frac{(i * j) * \operatorname{COM}(i, j)-\left(\mu_{x} * \mu_{y}\right)}{\sigma_{x} * \sigma_{y}}
$$

Where $\mu_{x}, \mu_{y}$ and $\sigma_{x}, \sigma_{y}$ are the mean and standard deviations of probability matrix GLCM along row wise $\mathrm{x}$ and column wise y

$$
\begin{aligned}
& C P=\sum_{i=1}^{m} \sum_{j=1}^{n}\left(i+j-\mu_{x}-\mu_{y}\right)^{4} * \operatorname{COM}(i, j) \\
& I M C 1=\sum_{i=1}^{m} \sum_{j=1}^{n} \frac{\log (i * j) * \operatorname{COM}((i, j)}{\mu_{x} * \mu_{y}}
\end{aligned}
$$

Where $P_{i j}$ is the pixel value of the image at position $(i, j)$ 


\section{RESULTS AND DISCUSSION}

The proposed DDRGRM model with CM features is implemented using a data set of 612 stone images collected from Mayang database, 678 stone images collected from VisTex database, 832 images collected from Paul Bourke database, 400 stone texture images collected from Google database i.e., a total of 2522 stone texture images. Sample images of each group are shown in Figure 9.

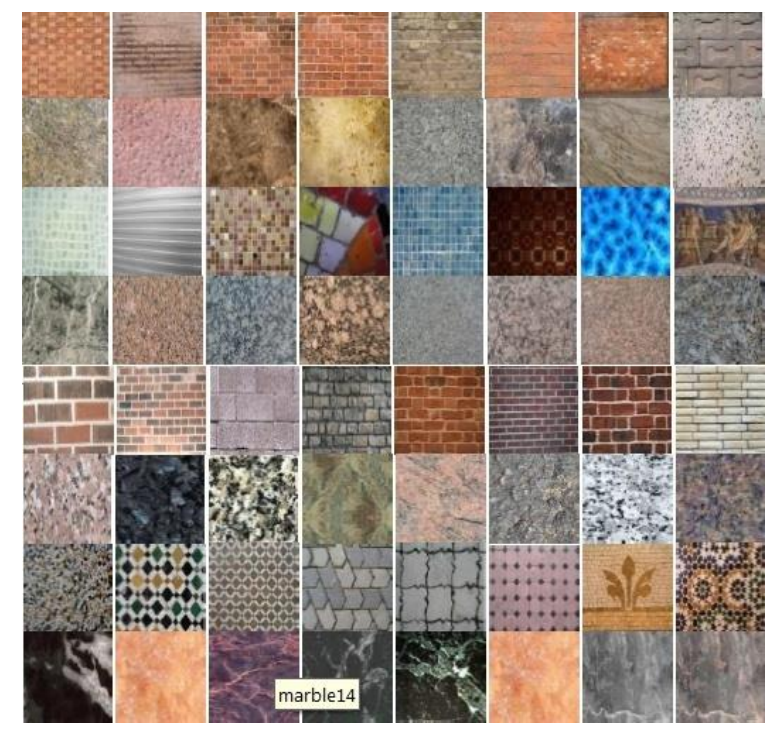

Figure 9. Sample stone texture images from various databases, 16 from each class

The three CM features i.e Correlation (CR), Cluster Prominence (CP) and Information measure of correlation1 (IMC1) are extracted on to the DDRGRM model of different stone texture groups of images and the results are stored in the feature vector. Feature set leads to representation of training images. The three $\mathrm{CM}$ features of stone images of four groups i.e. Marble, Granite, Bricks and Mosaic are shown in Tables 1, 2, 3 , and 4 respectively. Based on these feature set values the tested image is classified by using one of the two approaches and classified the stone images into one of the four pre-defined groups i.e., Marble, Granite, Bricks and Mosaic. The first approach uses the standard classification algorithms and second approach uses a user defined algorithm.

Table 1. Feature set values of the granite textures

\begin{tabular}{ccccc}
\hline \multirow{2}{*}{ Sno } & IMAGE & \multicolumn{3}{c}{ CM features on DDRGRM model } \\
\cline { 3 - 5 } & NAME & Correlation & Cluster Prominence & Information measure of correlation1 \\
\hline 1 & Granite001 & 0.0213 & 593 & 0.7563 \\
2 & Granite002 & 0.0356 & 613 & 2.36 \\
3 & Granite003 & 0.1021 & 601 & 13.91 \\
4 & Granite004 & 0.0564 & 487 & 12.36 \\
5 & Granite005 & 0.0967 & 476 & 10.35 \\
6 & Granite006 & 0.1063 & 513 & 9.36 \\
7 & Granite007 & 0.1007 & 576 & 8.64 \\
8 & Granite008 & 0.0965 & 519 & 7.49 \\
9 & Granite009 & 0.0640 & 412 & 6.76 \\
10 & Granite010 & 0.0402 & 472 & 2.42 \\
11 & Granite011 & 0.0508 & 396 & 4.36 \\
12 & Granite012 & 0.0197 & 386 & 0.66 \\
13 & Granite013 & 0.0231 & 393 & 0.99 \\
14 & Granite014 & 0.0937 & 592 & 11.78 \\
15 & Granite015 & 0.0941 & 553 & 12.37 \\
16 & Granite016 & 0.0452 & 412 & 2.35 \\
17 & Granite017 & 0.0733 & 402 & 8.97 \\
18 & Granite018 & 0.0828 & 497 & 10.16 \\
19 & Granite019 & 0.1070 & 778 & 13.08 \\
20 & Granite020 & 0.0354 & 712 & 1.3500 \\
\hline
\end{tabular}


Table 2. Feature set values of the bricks textures

\begin{tabular}{ccccc}
\hline \multirow{2}{*}{ Sno } & \multirow{2}{*}{ IMAGE NAME } & \multicolumn{3}{c}{ CM features on DDRGRM model } \\
\cline { 3 - 5 } & Correlation & Cluster Prominence & Information measure of correlation1 \\
\hline 1 & Brick001 & 0.2356 & 1122 & 0.596 \\
2 & Brick002 & 0.5686 & 1693 & 0.2988 \\
3 & Brick003 & 0.3568 & 1368 & 0.1235 \\
4 & Brick004 & 0.3286 & 1076 & 0.1135 \\
5 & Brick005 & 0.2288 & 804 & 0.0617 \\
6 & Brick006 & 0.3152 & 918 & 0.0865 \\
7 & Brick007 & 0.2964 & 1013 & 0.1025 \\
8 & Brick008 & 0.2564 & 1246 & 0.1135 \\
9 & Brick009 & 0.2737 & 1169 & 0.0750 \\
10 & Brick0010 & 0.3156 & 1175 & 0.1365 \\
11 & Brick0011 & 0.3643 & 1069 & 0.2165 \\
12 & Brick0012 & 0.3594 & 1521 & 0.2645 \\
13 & Brick0013 & 0.2476 & 1009 & 0.2135 \\
14 & Brick0014 & 0.2369 & 1035 & 0.1965 \\
15 & Brick0015 & 0.2200 & 1080 & 0.0491 \\
16 & Brick0016 & 0.2605 & 1212 & 0.0662 \\
17 & Brick0017 & 0.2658 & 1412 & 0.0655 \\
18 & Brick0018 & 0.2754 & 1124 & 0.0564 \\
19 & Brick0019 & 0.2341 & 1036 & 0.0574 \\
20 & Brick0020 & 0.2457 & 1217 & 0.1865 \\
\hline
\end{tabular}

Table 3. Feature set values of the mosaic textures

\begin{tabular}{ccccc}
\hline \multirow{2}{*}{ Sno } & \multirow{2}{*}{ IMAGE NAME } & \multicolumn{3}{c}{ CM features on DDRGRM model } \\
\cline { 3 - 5 } & Mosaic.001 & Correlation & Cluster Prominence & Information measure of correlation1 \\
\hline 1 & Mosaic.002 & 0.1768 & 897 & 0.0093 \\
3 & Mosaic.003 & 0.2013 & 1136 & 0.0165 \\
4 & Mosaic.004 & 0.1947 & 1037 & 0.0501 \\
5 & Mosaic.005 & 0.1822 & 967 & 0.0465 \\
6 & Mosaic.006 & 0.1232 & 813 & 0.0387 \\
7 & Mosaic.007 & 0.2159 & 811 & 0.0171 \\
8 & Mosaic.008 & 0.1885 & 830 & 0.0542 \\
9 & Mosaic.009 & 0.1936 & 907 & 0.0392 \\
10 & Mosaic.010 & 0.1395 & 814 & 0.0365 \\
11 & Mosaic.011 & 0.1265 & 889 & 0.0213 \\
12 & Mosaic.012 & 0.1364 & 914 & 0.0258 \\
13 & Mosaic.013 & 0.1457 & 923 & 0.0418 \\
14 & Mosaic.014 & 0.1568 & 854 & 0.0468 \\
15 & Mosaic.015 & 0.1356 & 872 & 0.0495 \\
16 & Mosaic.016 & 0.1508 & 963 & 0.0467 \\
17 & Mosaic.017 & 0.2153 & 998 & 0.0254 \\
18 & Mosaic.018 & 0.2052 & 947 & 0.0505 \\
19 & Mosaic.019 & 0.0822 & 865 & 0.0479 \\
20 & Mosaic.020 & 0.1664 & 809 & 0.0073 \\
\hline
\end{tabular}

Table 4. Feature set values of the marble textures

\begin{tabular}{ccccc}
\hline \multirow{2}{*}{ Sno } & \multirow{2}{*}{ IMAGE NAME } & \multicolumn{3}{c}{ GLCM features on DDRGRM model } \\
\cline { 3 - 5 } & & Correlation & Cluster Prominence & Information measure of correlation1 \\
\hline 1 & Marble.001 & 0.1957 & 441 & 57.18 \\
3 & Marble.002 & 0.1313 & 787 & 19.96 \\
3 & Marble.003 & 0.1267 & 613 & 20.56 \\
4 & Marble.004 & 0.1364 & 648 & 30.12 \\
5 & Marble.005 & 0.1463 & 513 & 21.13 \\
6 & Marble.006 & 0.1266 & 624 & 21.85 \\
7 & Marble.007 & 0.1235 & 529 & 22.83 \\
8 & Marble.008 & 0.1368 & 573 & 26.46 \\
9 & Marble.009 & 0.1299 & 613 & 24.20 \\
10 & Marble.010 & 0.1362 & 638 & 24.65 \\
11 & Marble.011 & 0.1472 & 679 & 25.68 \\
12 & Marble.012 & 0.1369 & 713 & 26.29 \\
13 & Marble.013 & 0.1458 & 752 & 27.49 \\
14 & Marble.014 & 0.1864 & 743 & 28.13 \\
15 & Marble.015 & 0.1765 & 481 & 30.15 \\
16 & Marble.016 & 0.1861 & 378 & 59.98 \\
17 & Marble.017 & 0.1957 & 381 & 34.99 \\
18 & Marble.018 & 0.1613 & 603 & 50.84 \\
19 & Marble.019 & 0.1823 & 423 & 42.18 \\
20 & Marble.020 & 0.1671 & 413 &
\end{tabular}




\subsection{By using Standard classification Algorithms}

The proposed method is tested using k-Nearest Neighbor Classifier (K-NNC) and support vector machines (SVM) are used for classification purpose. All experiments are carried out on a PC machine with i5 processor $2.6 \mathrm{GHz}$ CPU and 4 GB RAM memory under MATLAB 10.1a platform. $40 \%$ of the each database is used for training and remaining $60 \%$ images are used for testing purpose i.e. 1008 images are used for training purpose and 1514 images are used for testing purpose. The percentage of classification of the proposed method with K-NNC applied and generated values are listed out in Table 5. The percentage of classification of the proposed method with Support Vector Machine (SVM) applied and generated values are listed out in Table 6.

Table 5. Percentage of classification when k-NNC algorithm is applied

\begin{tabular}{lccccc}
\hline \multirow{2}{*}{ Texture Group } & \multicolumn{5}{c}{ Classification Rate of considered Stone texture Databases when k-NN classifier applied } \\
\cline { 2 - 6 } & VisTex & Mayang & Google & Paul Bourke & Overall \% \\
\hline Bricks & 95.9 & 95.76 & 95.98 & 96.35 & 96 \\
Marble & 95.94 & 96.34 & 96.06 & 96.04 & 96.1 \\
Granite & 95.99 & 95.76 & 96.28 & 96.35 & 96.1 \\
Mosaic & 95.88 & 96.02 & 96.52 & 95.3 & 95.93 \\
\hline
\end{tabular}

Table 6. Percentage of classification when SVM algorithm is applied

\begin{tabular}{lccccc}
\hline \multirow{2}{*}{ Texture Group } & \multicolumn{4}{c}{ Classification Rate of considered Stone texture Databases when SVM classifier applied } \\
\cline { 2 - 5 } & VisTex & Mayang & Google & Paul Bourke & Overall \% \\
\hline Bricks & 95.93 & 96.13 & 96.14 & 96.05 & 96.06 \\
Marble & 96.51 & 96.21 & 96.24 & 96.09 & 96.26 \\
Granite & 95.93 & 96.43 & 96.24 & 96.14 & 96.19 \\
Mosaic & 96.19 & 96.67 & 96.07 & 96.03 & 96.24 \\
\hline
\end{tabular}

From above two tables, it is observed that when the K-NN classifier applied to the proposed method obtained classification percentage as $96.03 \%$ and the classification percentage when SVM is applied is 96.19\%. Almost two classification algorithms gave same classification percentage and it is high. So the proposed DDRGRM model is well suited for extraction of features from stone images and to classify the stone textures into 4 groups.

\subsection{By using Standard classification Algorithms}

Based on the features extracted on the training data set, the proposed user defined approach derives a classification approach as shown in algorithm 1 to classify the stone textures into one of the four predefined groups. So as to test the efficiency of the user defined classification approach the test data set is collected randomly from different stone texture databases.

Algorithm 1: Algorithm for Classification of Stone textures into 4 pre-defined groups using CM feature on DDRGRM model of stone images.

Begin

if $\mathrm{CP}>800 \& \& \mathrm{CR}>=0.219$ then

Print (stone image age is classified as 'Bricks Class');

Else if $\mathrm{CP}>800 \& \& \mathrm{CR}<0.219$ then

Print (stone image age is classified as 'Mosaic Class');

Else if $\mathrm{CP}<800 \& \&$ IMC1 $>19$ then

Print (stone image age is classified as 'Marble Class');

Else if $\mathrm{CP}<800 \& \&$ IMC $1<19$ then

Print (stone image age is classified as 'Granite Class');

Else

Print (stone image age is classified as 'Unkonown Class');

End 
Table 7. Classification rates of stone images into 4 groups using CM feature on DDRGRM model of texture images based on Algorithm 1.

\begin{tabular}{llcccc}
\hline \multirow{2}{*}{$\begin{array}{c}\text { Texture } \\
\text { Group }\end{array}$} & \multicolumn{5}{c}{ Classification Rate of considered Stone texture Databases when User define Classification Algorithm is used } \\
\cline { 2 - 5 } & VisTex & Mayang & Google & Paul Bourke & Overall \% \\
\hline Bricks & 95.97 & 96 & 96.11 & 96.25 & 96.08 \\
Marble & 96.28 & 96.33 & 96.2 & 96.12 & 96.23 \\
Granite & 96.01 & 96.15 & 96.31 & 96.3 & 96.19 \\
Mosaic & 96.09 & 96.4 & 96.35 & 95.72 & 96.14 \\
\hline
\end{tabular}

From the two sections, observe that the extracted features are well suited for classifiation of stone textures when standard and user defined classification algorithms. For analysing the results the confsion matrix is generated when user defined algorithm is applied on test database. The confusion matrix is shown in Table 8. The confusion matrix shows the classified class for each input texture in test database.

Table 8. Confusion matrix of the proposed method

\begin{tabular}{ccccc}
\hline Texture Group & Marble & Mosaic & Bricks & Granite \\
\hline Marble & 632 & 2 & 1 & 1 \\
Mosaic & 0 & 624 & 1 & 1 \\
Bricks & 2 & 1 & 624 & 2 \\
Granite & 1 & 2 & 0 & 626 \\
\hline
\end{tabular}

\section{COMPARISON WITH OTHER EXISTING METHODS:}

The proposed approach based on GLCM highlight on DDRGRM for stone texture classification has shown better classification rate in comparison with other existing approaches. The results of other existing approaches that are considered for comparison include: classification approach proposed by Vijay et al [22] which used Overlapped 5-bit T-Patterns Occurrence on 5-by-5 sub images, Wavelet based Histogram on Texton Patterns (WHTP) [23] proposed by Sasi Kiran et al, texture classification based on Texton Features [24] by Ravi babu et al and approach based on Syntactic Pattern on 3D technique [25]. It is quite evident that, the proposed strategy resulted in high characterization rate than the existing techniques. The classification rate for the proposed and other existing strategies are shown in Table 9 and the same was portrayed using graphical representation in Figure 10.

Table 9. Percentage mean classification rates for proposed DDRGRM model and other existing methods in

\begin{tabular}{|c|c|c|c|c|c|}
\hline Image Database & $\begin{array}{l}\text { 5-bit 'T' Pattern } \\
\text { Approach }\end{array}$ & $\begin{array}{l}\text { Syntactic Pattern } \\
\text { on 3D method }\end{array}$ & $\begin{array}{l}\text { Texton Feature } \\
\text { Detection }\end{array}$ & $\begin{array}{c}\text { Wavelet based Histogram } \\
\text { on Texton Patterns }\end{array}$ & $\begin{array}{c}\text { Proposed } \\
\text { DDRGRM Method }\end{array}$ \\
\hline VisTex & 95.95 & 93.15 & 95.46 & 92.87 & 95.93 \\
\hline $\begin{array}{c}\text { Texture Images Taken } \\
\text { by Camera }\end{array}$ & 96.35 & 92.87 & 95.12 & 91.7 & 96.85 \\
\hline Google & 96.76 & 93.32 & 94.86 & 93.56 & 96.96 \\
\hline Mayang & 95.85 & 92.83 & 94.39 & 92.95 & 96.15 \\
\hline Paul Bourke & 95.93 & 93.05 & 95.23 & 93.05 & 95.98 \\
\hline
\end{tabular}

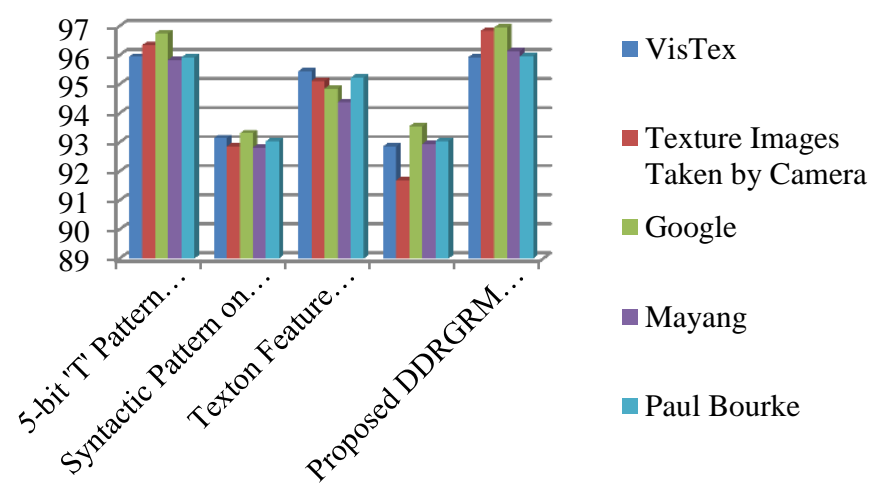

Figure 10. Comparison graph showing the classification rate of proposed and other existing approaches For different data sets 


\section{CONCLUSION}

The proposed DDRGRM strategy utilizing CM characterized stone textures into four groups by means of dimensionality reduction and reduced gray level of the texture images. Still the proposed approach achieved high classification rate, by retaining all critical nearby components including edge highlights and using three important Haralick parameters for powerful exact stone surface grouping. The proposed technique definitely lessened the computational time due to reduced dimensionality and gray level. Further the proposed approach extracted the features which are suitable to apply both existing standard classification approaches like k-NN and SVM approaches and also the user defined approach. This helped in verifying the efficiency of the proposed DDRGRM approach. It is evident from above results that proposed approach has resulted in high classification accuracy of $96.37 \%$ in comparison with $96.03 \%$ and $96.19 \%$ by k-NN classification and SVM approaches.

\section{REFERENCES}

[1] S. Arivazhagan and L. Ganesan, "Texture segmentation using wavelet transform," Pattern Recognition Letters, vol. 24, no. 16, pp. 3197-3203, 2003.

[2] Kaplan, L. M., "Extended fractal analysis for texture classification and segmentation," IEEE Transactions on Image Processing, vol. 8, no. 11, pp. 1572-1585, 1999.

[3] P. P. Raghu and B. Yegnanarayana, "Segmentation of gabor filtered textures using deterministic relaxation,"IEEE Transactions on Image Processing, vol. 5, no. 12, pp. 1625-1636, 1996.

[4] T. Chang and Kuo, C. C. J., "Texture analysis and classification with tree-structured wavelet transform," IEEE Trans. Image Processing, Vol 2, no. 4, pp. 429-442, 1993.

[5] J. L. Chen and Kundu, "Unsupervised texture segmentation using multi-channel decomposition and hidden Markov models," IEEE Trans. Image. Processing, Vol. 4, no. 5, pp. 603-620, 1995.

[6] R. W. Conners, "Toward a set of statistical features which measure visually perceivable qualities of texture," in Proc. Pattern Recognition Image Processing Conf., pp. 382-390, 1979.

[7] Bovik, A. C., Clark, M. and Geisler, W. S. "Multichannel texture analysis using localized spatial filters", IEEE Trans. Patt. Anal. Mach. Intell., vol. 12, no. 1, pp. 55-73, 1990.

[8] R.M. Haralick, K. Shanmugam, I. Dinstein, "Texture features for image classification", IEEE Transactions on System Man Cybernet, Vol. 8, no. 6, pp. 610-621, 1973.

[9] V Vijaya Kumar, et.al., " A New Method of Texture Classification using various Wavelet Transforms based on Primitive Patterns", ICGST International Journal on Graphics, Vision and Image Processing, GVIP, Vol.8, Issue 2, pp. 21-27, 2008

[10] A Yuniarti, "Classification and numbering of dental radiographs for an automated human identification system," TELKOMNIKA Telecommunication, Computing, Electronics and Control., vol. 10, no. 1, pp. 137-146, 2012.

[11] Haralick, R. M. "Statistical and structural approaches to texture", 4th Int. Joint Conf. Pattern Recognition, pp. 4560, 1979.

[12] V. V. Krishna, et al., "Classification of textures based on distance function of linear patterns using mathematical morphology," Proceedings of ICEM, conducted by JNT University, India, 2005.

[13] Vijaya Kumar, V. et al., "An Innovative Technique of Texture Classification and Comparison Based on Long Linear Patterns," Journal of Comp. Science, vol. 3, no.8, pp. 633-638, 2007.

[14] IKGD Putra, E Erdiawan, "High performance palmprint identification system based on two dimensional gabor," TELKOMNIKA Telecommunication Computing Electronics and Control., vol. 8, no. 3, pp. 309-318, 2010.

[15] V. V. Kumar, et al., "Classification of Textures by Avoiding Complex Patterns, Science publications," Journal of Computer Science, 2008.

[16] V. V. Kumar, et al., "A measure of patterns trends on various types of preprocessed images," International Journal of Computer Science and Network Security, vol/issue: 7(8), pp. 253-257, 2007.

[17] U. S. N. Raju, et al., "Texture Description using Different Wavelet Transforms Based on Statistical Parameters," proceedings of the 2nd International Symposium on Wavelets Theory \& Applications in Applied Mathematics, Signal Processing \& Modern Science (WAV '08), Istanbul, Turkey, pp. 174-178, 2008.

[18] Juan Wang, Jiangshe Zhang, and Jie Zhao," Texture Classification Using Scattering Statistical and Co-occurrence Features", Mathematical Problems in Engineering, vol. 2016, Article ID 3946312, 6 pages, 2016.

[19] Dr. D.Siva Kumar, "Stone Texture Classification and Discrimination by Edge Direction Movement", International Journal of Innovative Research in Computer and Communication Engineering, Vol. 4, no. 4, pp:8057-8063, 2016.

[20] Ratna Bhargavi V, Ranjan K. Senapati, "Bright Lesion Detection in Color Fundus Images Based on Texture Features", Bulletin of Electrical Engineering and Informatics , Vol. 5, No. 1, March 2016, pp. 92 100, DOI: 10.11591/eei.v5i1.553

[21] Xiaorong Xue, Liping Di, Liying Guo, and Li Lin,"Unsupervised Classification of Fully Polarimetric SAR Image Based on Polarimetric Features and Spatial Features", TELKOMNIKA, Vol.14, No.3A, September 2016, pp. 244 251

[22] Vijay Kumar, Palnati, SVVSR Kumar, Pullela.,, Nakkella Madhuri, M Uma Devi, "Stone Image Classification Based on Overlapped 5-bit T-Patterns Occurrence on 5-by-5 Sub Images", International Journal of Electrical and Computer Engineering (IJECE), Vol. 6, no. 3, pp. 1152 -1160, ,June 2016. 
[23] Sasi Kiran, Jangala., Ravi Babu, U., and Dr. V V Kumar "Wavelet based Histogram method for classification of textures", IJECT, Volume: 4, issue: 3, pages: 149-164, 2013.

[24] Ravi Babu U , Vijaya Kumar, Sujatha B, "Texture Classification Based on Texton Features", I.J. Image, Graphics and Signal Processing, Vol. 4, no. 8, pages: 36-42, 2012.

[25] A Suresh and V. Vijaya Kumar, "Pattern Based Classification of Stone Textures on a Cubical Mask," International Journal of Univ. Comp. Sci., Vol.1, no.1, pp. 4-9, 2010. 